\title{
Paniculitis en pancreatitis aguda. Reporte de un caso y revisión de la literatura
}

\author{
Rómulo Vargas, ${ }^{1}$ Alan Ovalle Hernández, ${ }^{2}$ Daniela Cáceres Escobar, ${ }^{3}$ Oscar Lucero Pantoja ${ }^{3}$ \\ ${ }^{1}$ Profesor de Medicina interna. Jefe de la unidad de Gastroenterología. \\ ${ }^{2}$ Médico internista y gastroenterólogo. Profesor de la unidad de Gastroenterología. \\ ${ }^{3}$ Médica internista. Fellow de Gastroenterología. \\ ${ }^{4}$ Residente de Medicina interna. \\ Unidad de Gastroenterología. Pontificia Universidad Javeriana, Hospital Universitario San Ignacio, Bogotá, Colombia.
}

Acta Gastroenterol Latinoam 2021;51(2):223-227

Recibido: 23/06/2020 / Aceptado: 10/05/2021 / Publicado online: 21/06/2021 / https://doi.org/10.52787/ihbj3544

\begin{abstract}
Resumen
La paniculitis pancreática es una manifestación cutánea que se puede presentar como una complicación de la pancreatitis aguda. Se presenta el caso de un paciente que cursó una pancreatitis aguda inicialmente clasificada como leve, pero que, en el transcurso de la enfermedad, se asoció a la presencia de una exacerbación de dolor junto con lesiones en el dorso eritematosas, dolorosas y bien definidas. En el control tomográfico, se corroboró la presencia de la paniculitis y necrosis peripancreática, con gas periportal. El paciente cursó una descompensación clinica asociada a la sospecha del sindrome compartimental, por lo que se decidió realizar una laparotomía exploratoria. La presentación de paniculitis pancreática es una manifestación poco frecuente, por este motivo consideramos pertinente la descripción de este caso y su revisión en la literatura.
\end{abstract}

Palabras claves. Paniculitis pancreática, pancreatitis aguda, reporte de caso.

Correspondencia: Rómulo Vargas Cra 7 No 40-62. Bogotá, Colombia Correo electrónico: rdvargas@husi.org.co

\section{Panniculitis in Acute Pancreatitis. A Case Report and Literature Review}

\section{Summary}

Pancreatic panniculitis is a cutaneous manifestation that can occur as a complication of acute pancreatitis. We present the case of a patient who had an acute pancreatitis initially classified as mild, but, during the course of the disease, was associated with the presence of an exacerbation of pain together with erythematous, painful and well-defined dorsal lesions. In the tomographic control, the presence of the panniculitis and peri-pancreatic necrosis with periportal gas was confirmed. The patient had a clinical decompensation associated with the suspicion of compartment syndrome. For this reason, it was decided to perform an exploratory laparotomy. The presentation of pancreatic paniculitis is an infrequent manifestation, thus we consider pertinent the description of this case and its review in the literature.

Keywords. Pancreatic panniculitis, acute pancreatitis, case report.

\section{Introducción}

La paniculitis pancreática es una forma poco frecuente de necrosis grasa subcutánea asociada con el proceso inflamatorio del parénquima pancreático. Se manifiesta hasta en el $2 \%$ de los casos de pancreatitis, especialmente en la pancreatitis severa. ${ }^{1}$ Usualmente, se describe en asociación a enfermedades pancreáticas benignas, como la pancreatitis aguda o la crónica y, de forma menos común, con un carcinoma. ${ }^{2}$ Hasta la fecha, hay un número 
limitado de publicaciones asociadas a esta manifestación, la mayoría de España, con series de no más de 7 casos. ${ }^{1-3}$ En Colombia, no se ha reportado esta patología.

Aunque el mecanismo no está claro, se cree que está asociada a la necrosis tisular generada por los altos niveles en suero de enzimas pancreáticas como la lipasa, la amilasa y la tripsina. ${ }^{4}$

El tratamiento se basa en el control del proceso inflamatorio pancreático, por lo que el inicio de la antibióticoterapia no ha demostrado beneficio. ${ }^{5}$ En la mayoría de los casos, el pronóstico es benigno, aunque en pocas ocasiones se puede asociar a una patología neoplásica pancreática. ${ }^{6}$

\section{Caso clínico}

El paciente de 84 años, hipertenso en tratamiento con losartán y con dislipidemia mixta (hipercolesterolemia e hipertrigliceridemia) sin manejo farmacológico, acudió al servicio de urgencias por un dolor de tres días en el epigastrio y en el hipocondrio derecho, irradiado a la región dorsal, asociado a náuseas y episodios eméticos. En el momento del ingreso, está álgico, taquicárdico, afebril, sin desaturación ni taquipnea. En el examen físico, está con ictericia en las escleras, dolor en la palpación en el hipocondrio derecho y sin signos de irritación peritoneal. Los paraclínicos del ingreso se presentan sin leucocitosis ni neutrofilia, el perfil hepático está alterado con un patrón colestásico y hay una elevación de la amilasa e hiperlactatemia (Tabla 1).

Se realizó un estudio ecográfico. Se observó evidencia de barro biliar en el cuello vesicular, la dilatación de la vía biliar intra y extrahepática, con un diámetro de colédoco de $12 \mathrm{~mm}$ y barro biliar en su interior. Adicionalmente, el páncreas estaba aumentado de tamaño con líquido peripancreático.

Se la consideró como una pancreatitis aguda de origen biliar por los criterios clínicos, serológicos e imagenológicos, moderadamente severa por la clasificación de Atlanta. Se descartó la causa farmacológica, asociada a una hipertrigliceridemia, una hipercalcemia o por el consumo crónico de alcohol. Se inició el tratamiento con líquidos endovenosos isotónicos a $5 \mathrm{ml} / \mathrm{kg} / \mathrm{h}$, durante las primeras 24 horas, junto con el manejo analgésico. Los paraclínicos de control mostraron un descenso de las bilirrubinas y adecuada depuración del lactato, por lo que se planteó la posibilidad de un cálculo biliar transitado. Presentó glucometrías fuera de rango, con HbA1c de $12 \%$, por lo cual se inició el manejo con insulinas en el contexto de diabetes mellitus de novo. Se reinició el tratamiento vía oral dentro de las primeras 24 horas sin complicaciones.
Tabla 1. Paraclínicos del ingreso

\begin{tabular}{|c|c|c|}
\hline Paraclínico & Resultado & Valor de referencia \\
\hline Leucocitos & $8,1 \times 10^{3} \mathrm{cel} / \mathrm{uL}$ & $4,5-10 \times 10^{3} \mathrm{cel} / \mathrm{uL}$ \\
\hline Hemoglobina & $13,3 \mathrm{~g} / \mathrm{dL}$ & $13,5-18 \mathrm{~g} / \mathrm{dL}$ \\
\hline Hematocrito & $39,8 \%$ & $42-52 \%$ \\
\hline Plaquetas & $319,1 \times 10^{3} \mathrm{cel} / \mathrm{uL}$ & $150-450 \mathrm{cel} / \mathrm{uL}$ \\
\hline Neutrófilos & $7,6 \times 10^{3} \mathrm{cel} / \mathrm{uL}$ & $2,1-6,1 \times 10^{3} \mathrm{cel} / \mathrm{uL}$ \\
\hline Creatinina & $0,9 \mathrm{mg} / \mathrm{dL}$ & $0,72-1,18 \mathrm{mg} / \mathrm{dL}$ \\
\hline Nitrógeno ureico & $22,3 \mathrm{mg} / \mathrm{dL}$ & $7-25 \mathrm{~m} / \mathrm{dL}$ \\
\hline Sodio & $131 \mathrm{mmol} / \mathrm{L}$ & $136-146 \mathrm{mmol} / \mathrm{L}$ \\
\hline Potasio & $4 \mathrm{mmol} / \mathrm{L}$ & 3,5-5,1 mmol/L \\
\hline Calcio sérico (corregido) & $9,1 \mathrm{mg} / \mathrm{dL}$ & 8,5 a $10,2 \mathrm{mg} / \mathrm{dL}$ \\
\hline Proteína C reactiva & $6,19 \mathrm{mg} / \mathrm{dL}$ & $0-0,5 \mathrm{mg} / \mathrm{dL}$ \\
\hline Amilasa & $1215 \mathrm{u} / \mathrm{L}$ & $22-80 \mathrm{u} / \mathrm{L}$ \\
\hline Bilirrubina total & $4 \mathrm{mg} / \mathrm{dL}$ & $0,3-1 \mathrm{mg} / \mathrm{dL}$ \\
\hline Bilirrubina directa & $2,63 \mathrm{mg} / \mathrm{dL}$ & $0-0,2 \mathrm{mg} / \mathrm{dL}$ \\
\hline Bilirrubina indirecta & $1,37 \mathrm{mg} / \mathrm{dL}$ & $0-1,1 \mathrm{mg} / \mathrm{dL}$ \\
\hline Fosfatasa alcalina & 198 u/L & 30-120 u/L \\
\hline Aspartato aminotransferasa & $657 \mathrm{u} / \mathrm{L}$ & $0-50 \mathrm{u} / \mathrm{L}$ \\
\hline Alanino aminotransferasa & $458 \mathrm{u} / \mathrm{L}$ & $0-50 \mathrm{u} / \mathrm{L}$ \\
\hline Triglicéridos & $160 \mathrm{mg} / \mathrm{dL}$ & $10-150 \mathrm{mg} / \mathrm{dL}$ \\
\hline Colesterol total & $216 \mathrm{mg} / \mathrm{dL}$ & Menor a 200 mg/dL \\
\hline Lactato sérico & $3,01 \mathrm{mmol} / \mathrm{L}$ & $0-1,3 \mathrm{mmol} / \mathrm{L}$ \\
\hline $\mathrm{pH}$ arterial & 7,446 & $7,35-7,45$ \\
\hline $\mathrm{PCO}_{2}$ arterial & $26,2 \mathrm{mmHg}$ & $35-45 \mathrm{mmHg}$ \\
\hline $\mathrm{PO}_{2}$ arterial & $68,3 \mathrm{mmHg}$ & 80-100 mmHg \\
\hline $\mathrm{HCO}_{3}$ arterial & $17,6 \mathrm{mmol} / \mathrm{L}$ & $22-26 \mathrm{mmol} / \mathrm{L}$ \\
\hline Base exceso arterial & $-4,3 \mathrm{mmol} / \mathrm{L}$ & -2 a $2 \mathrm{mmol} / \mathrm{L}$ \\
\hline
\end{tabular}

En el cuarto día de hospitalización, el paciente presentó la reaparición del dolor abdominal asociado a signos inflamatorios locales dados por un eritema bien delimitado a nivel de los flancos (Figura 1). 
Figura 1. Paniculitis. Hay lesiones maculares eritematosas de bordes irregulares bien delimitadas, asociadas a un edema, con un diámetro aproximado de $35 \mathrm{~cm}$ en los flancos.
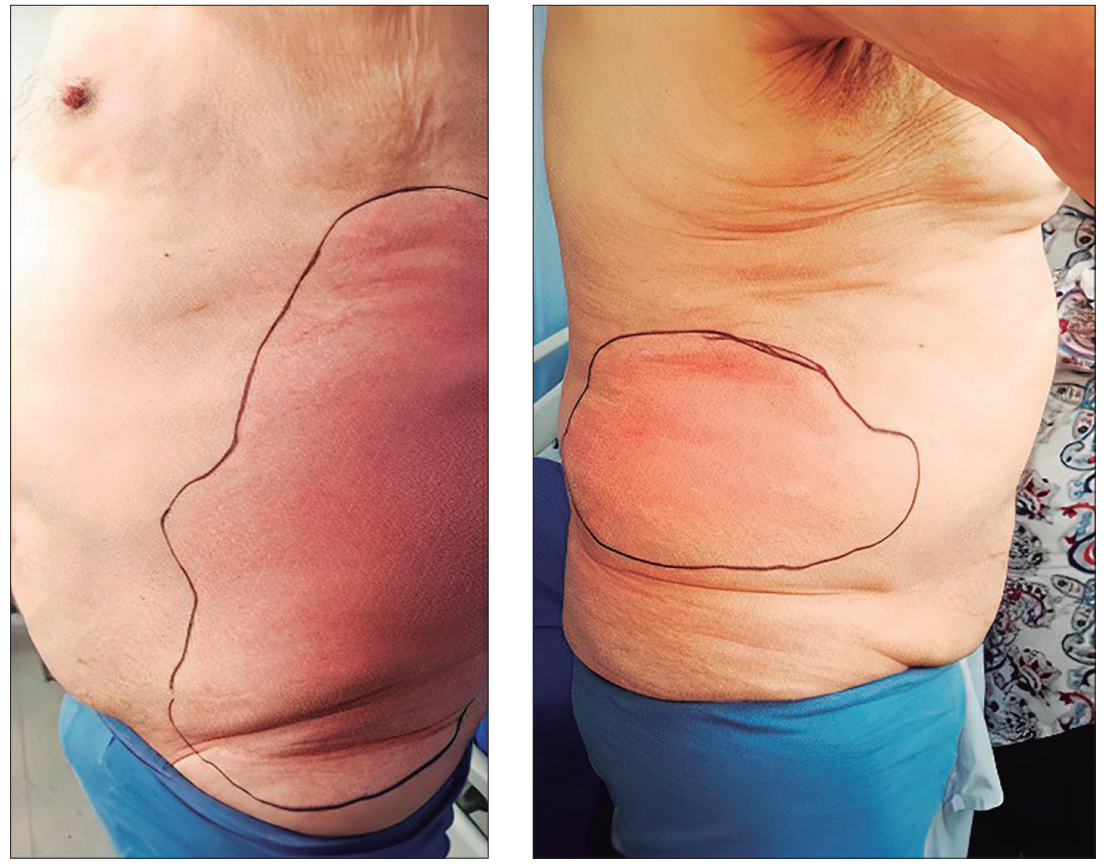

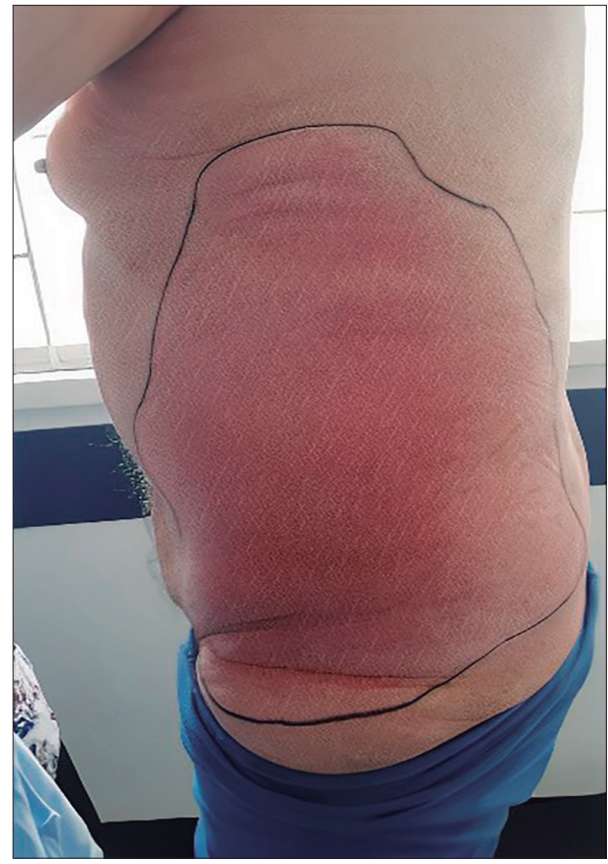

Se le solicitó una tomografía axial computarizada (TAC) del abdomen contrastado, con el fin de descartar complicaciones asociadas a la pancreatitis, y se reportó un aumento en el tamaño de la cabeza y el cuello del páncreas, con hiperdensidad y estriación de la grasa mesentérica peripancreática, asociado a líquido y gas peripancreático y periportal. Las vías biliares intra y extrahepática tenían calibre y curso normales para su edad. Adicionalmente, presentaba un edema de los tejidos blandos de la región lumbar izquierda (Figura 2). Los hallazgos fueron compatibles con una pancreatitis aguda necrosante peripancreática con una colección necrótica aguda y una paniculitis.

El paciente presentó un deterioro clínico asociado a un dolor abdominal no modulado, oliguria y la elevación de los azoados que obligó a hacer el diagnóstico diferencial de hipoperfusión, en el escenario de una hipertensión intraabdominal. Se le solicitó la toma de presión intraabdominal, a través de una sonda vesical con un valor de 22 $\mathrm{mmHg}$, por lo que se consideró posible una disfunción renal asociada al síndrome compartimental. Se le indicó el paso de la sonda nasogástrica y el inicio del tratamiento proquinético con metoclopramida sin mejoría, por lo que se decidió llevarlo a una laparotomía exploratoria que evidenció el engrosamiento del epiplón mayor en relación con unos focos de esteatonecrosis, presencia de focos de necrosis grasa en el retroperitoneo y páncreas con una colección de $30 \mathrm{cc}$ que fue drenada. Se exploró la vesícula biliar y se encontraron paredes engrosadas, con barro bi-
Figura 2. Se observa, en la TAC de abdomen contrastado, el aumento del tamaño de la cabeza y el cuello del páncreas (punta de flecha), con el incremento en la densidad y estriación de la grasa mesentérica peripancreática ( $A$ y C). Hay líquido y escaso gas peripancreático adyacente a la cabeza y extendiéndose al hilio hepático (C). El proceso inflamatorio descrito rodea la vena porta y la arteria hepática propia que se encuentran permeables y sin pseudoaneurismas. Se reconoce un edema de los tejidos blandos de la región lumbar izquierda (flechas). Se observa un derrame pleural bilateral (B).
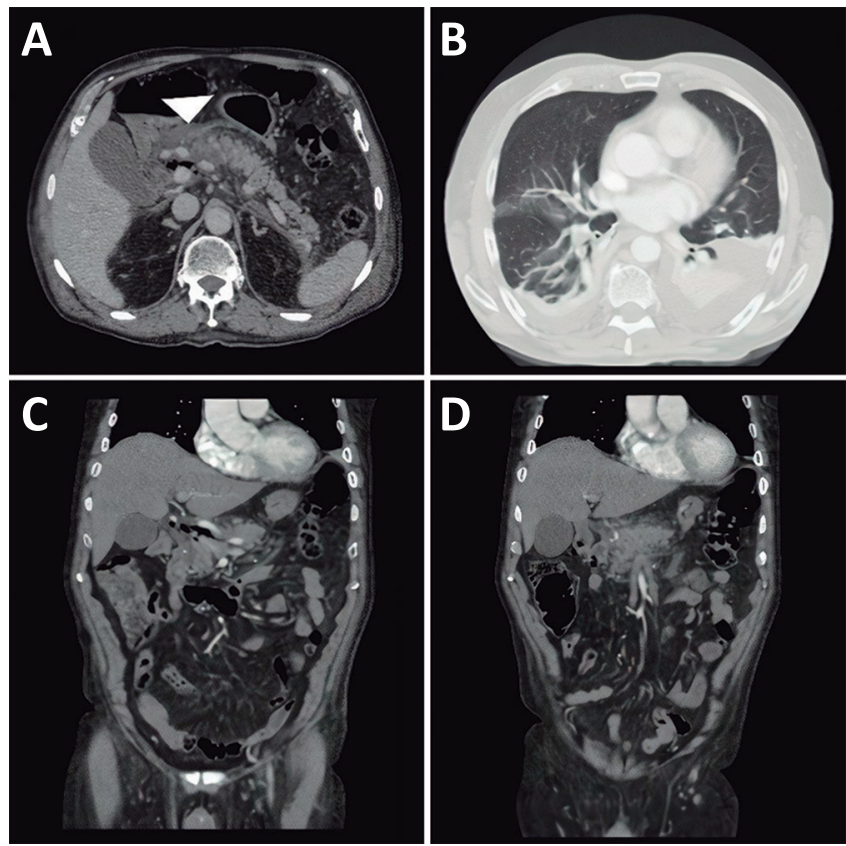
liar y cálculos, por lo que se le realizó una colecistectomía. No se evidenció una isquemia de las asas.

En los días siguientes al desbridamiento y lavado retroperitoneal bilateral, el paciente evolucionó de manera satisfactoria con mejoría de los parámetros hemodinámicos, la resolución de la lesión renal y de los cambios inflamatorios locales. Se le dio egreso al décimo día posoperatorio (Figura 3).

Figura 3. Evolución de las lesiones en la piel al cuarto 4 día posoperatorio.
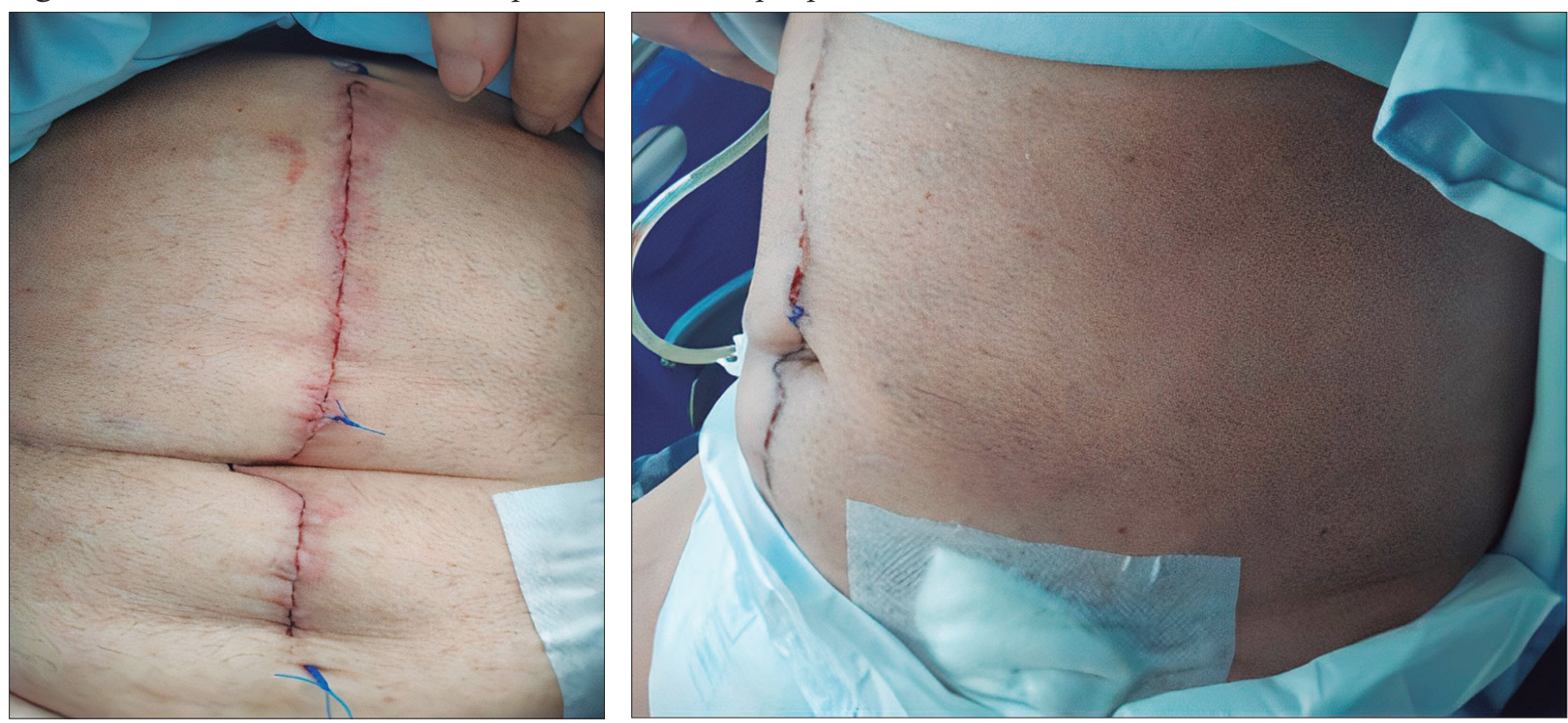

\section{Discusión}

La paniculitis pancreática es una manifestación infrecuente de la pancreatitis. Se presenta en menos del 2\% de los casos. ${ }^{1}$ Constituye una forma de necrosis grasa subcutánea asociada a la enfermedad pancreática. ${ }^{3}$ Es más frecuente en los hombres, con una edad entre 40 y 60 años, y se ha observado una relación con el alcoholismo crónico. La primera descripción de esta entidad data de 1947 , cuando el médico inglés Auger describió un paciente con lesiones típicas de paniculitis, a quien posteriormente se le diagnosticó un carcinoma de células acinares. ${ }^{4}$ No es una entidad exclusiva de la pancreatitis aguda, porque puede ocurrir durante la evolución de otras enfermedades pancreáticas primarias, ${ }^{5}$ y está, en la mayoría de las ocasiones, asociada con patologías pancreáticas benignas. En una menor proporción, se correlaciona con un carcinoma pancreático, ${ }^{6}$ un carcinoma neuroendocrino y un insulinoma. En una serie española de casos, la paniculitis pancreática fue la primera manifestación del adenocarcinoma de ampolla. ${ }^{7}$ Las lesiones vasculares peripancreáticas también deben considerarse dentro del diagnóstico diferencial de la paniculitis pancreática. ${ }^{8}$ De manera esporádica, se la ha vinculado como una manifestación de rechazo postrasplante, principalmente en el trasplante simultáneo de páncreas y riñón. ${ }^{9}$
La patogénesis no se conoce con claridad, aunque se cree que está asociada a la necrosis tisular generada por los altos niveles en suero de las enzimas pancreáticas como la lipasa, la amilasa y la tripsina.

Se presenta clínicamente como lesiones eritematosas, dolorosas y bien delimitadas; en ocasiones, compromete la región abdominal, aunque también se puede manifestar como nódulos subcutáneos eritematosos en los miembros inferiores, predominantemente sobre las rodillas y los tobillos, que se pueden semejar a un eritema nudoso. Puede presentarse conjuntamente con las artralgias secundarias a la necrosis grasa intraósea, ${ }^{9}$ configurando el síndrome de PPP (pancreatitis, paniculitis y poliartritis), que es una complicación extremadamente rara y está asociada con un pobre pronóstico. ${ }^{10} \mathrm{La}$ combinación de la necrosis grasa subcutánea, la poliartritis y la eosinofilia, en los pacientes con cáncer pancreático, es conocida como la triada de Schid e implica un mal pronóstico de sobrevivencia. ${ }^{11}$

En ocasiones, la paniculitis progresa y puede llegar a ulcerase, drenando contenido graso, con altos niveles de amilasa, lipasa y ácidos grasos. A nivel histológico, se puede observar la paniculitis lobular, sin vasculitis, con necrosis grasa y la presencia de adipocitos que pierden su núcleo y contienen depósitos granulares de calcio como resultado de la saponificación, llamados "adipocitos fantasmas". ${ }^{12}$ 
Estos hallazgos son muy específicos, pero no son patognomónicos, dado que otras patologías pueden generarlos, principalmente las infecciones por hongos que producen una alta carga de lipasa extracelular, como la aspergilosis subcutánea y la mucormicosis. ${ }^{12}$ Los paraclínicos, en la paniculitis pancreática, pueden mostrar leucocitosis y el aumento de los reactantes de fase aguda. También, se ha descrito la eosinofilia y la reacción leucemoide. ${ }^{13}$

El tratamiento de la paniculitis se ocupa del control de la enfermedad de base. ${ }^{7}$ A medida que las lesiones mejoran, disminuye la inflamación pancreática. No se ha descrito el beneficio de iniciar la antibióticoterapia en esta patología. ${ }^{8}$ En el caso de la neoplasia, es probable la recurrencia del cuadro, la progresión y ulceración de las lesiones en la piel. ${ }^{8}$

Finalmente, el manejo quirúrgico no está recomendado dentro del tratamiento de la pancreatitis aguda salvo en algunas excepciones, ${ }^{14}$ dado a que está asociado a una mayor morbilidad y mortalidad. En nuestro caso, la presencia de la hipertensión intraabdominal (presión mayor a $12 \mathrm{mmHg}$ ), junto con los signos sugestivos del síndrome compartimental abdominal, que no mejoraron a pesar de las medidas de descompresión con sonda nasogástrica y los agentes proquinéticos, llevaron a considerar la realización de la laparotomía. No obstante, para la mayoría de las complicaciones asociadas a la pancreatitis aguda, se busca un manejo no invasivo y, en caso de requerirse, se prefiere un tratamiento escalonado (también llamado step-up approach), donde se consideran inicialmente medidas endoscópicas o percutáneas seguidas por un desbridamiento mediante métodos mínimamente invasivos. ${ }^{14}$

\section{Conclusión}

La paniculitis pancreática es una manifestación poco frecuente de la enfermedad pancreática y, en la mayoría de las ocasiones, se asocia a patologías benignas. En los casos de pancreatitis aguda, su hallazgo debe hacer sospechar sobre la presencia de complicaciones locales: colecciones agudas (peri) pancreáticas o colección necrótica aguda. Su manejo consiste en el abordaje de la enfermedad pancreática de base y estará reflejado en la resolución de los síntomas inflamatorios. Este hallazgo le permite al médico clínico sospechar las complicaciones asociadas a una pancreatitis severa que posibiliten realizar conductas tempranas y mejorar el desenlace.

Agradecimientos. Al servicio de Gastroenterología del Hospital Universitario San Ignacio por su colaboración en la realización del artículo.

Sostén financiero. No se recibió el apoyo de becas u otros aportes económicos recibidos para la realización del estudio.

\section{Aviso de derechos de autor}

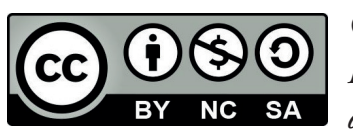

(C) 2021 Acta Gastroenterológica Latinoamericana. Este es un artículo de acceso abierto publicado bajo los términos de la Licencia Creative Commons Attribution (CC BY-NC-SA 4.0), la cual permite el uso, la distribución $y$ la reproducción de forma no comercial, siempre que se cite al autor y la fuente original.

Cite este artículo como: Vargas $R$, Ovalle Hernández A, Cáceres Escobar D y col. Paniculitis en pancreatitis aguda. Reporte de un caso y revisión de la literatura. Acta Gastroenterol Latinoam. 2021;51(2):223-7. https://doi.org/10.52787/ ihbj3544

\section{Referencias}

1. Ballester Sánchez R, Bustos BU, Hernández Bel P, Torrijos Aguilar A, Miquel VA De. Paniculitis pancreática. Revisión de 7 casos. Piel: formación continua e dermatología. 2012;27(7):367-71.

2. Segurado Rodríguez A, Guerra Tapia A, Jaén Olasolo P, Cueva Santos J. Paniculitis pancreática: estudio de 12 casos y valoración comparativa de sus caracteres epidemiológicos, clínicos, histopatológicos y terapéuticos. Actas Dermosifiliogr. 1999;90:227-34.

3. Requena L, Sánchez Yus E. Panniculitis. Part II. Mostly lobular panniculitis. J Am Acad Dermatol. 2001;45(3):325-61.

4. Torres-Navarro I, Rojas-Ferrer N, Botella-Estrada R. Pancreatic panniculitis. Rev Esp Enferm Dig. 2019;111(10):812-3.

5. Graham PM, Altman DA, Gildenberg SR. Panniculitis, pancreatitis, and polyarthritis: a rare clinical syndrome. Cutis. 2018;101(1):E34-7.

6. Blauvelt $\mathrm{H}$. A case of acute pancreatitis with subcutaneous fat necrosis. Br J Surg. 1946;34(134):207-8.

7. Jo S, Song S. Pancreatitis, panniculitis, and polyarthritis syndrome caused by pancreatic pseudocyst: A case report. World J Clin Cases. 2019;7(18):2808-14.

8. Miulescu R, Vasile Balaban D, Sandru F, Jinga M. Cutaneous Manifestations in Pancreatic Diseases - A Review. J Clin Med. 2020;9(8):2611.

9. Moro M, Moletta L, Blandamura S, Sperti C. Acinar cell carcinoma of the pancreas associated with subcutaneous panniculitis. JOP. 2011;12(3):292-6.

10. Beveridge M, Pei S, Tsoukas MM. Pancreatic panniculitis in a pancreas-kidney transplant patient resolved after immunosuppression increase: Case report and review of literature. JAAD Case Reports. 2015;1(2):101-5.

11. Requena L, Sitthinamsuwan P, Santonja C, Fernández-Figueras MT, Rodríguez-Peralto JL, Argenyi Z, et al. Cutaneous and mucosal mucormycosis mimicking pancreatic panniculitis and gouty panniculitis. J Am Acad Dermatol. 2012;66(6):975-84.

12. Evans AC, Singhi AD, Zeh HJ, Bahary N, Brand RE. An Unexpected Etiology of Pancreatic Panniculitis: A Case Report. J Pancreat Cancer. 2017;3(1):1-4.

13. Fernández-Sartorio C, Combalia A, Ferrando J, Alsina M, Iranzo P, Estrach T, et al. Pancreatic panniculitis: A case series from a tertiary university hospital in Spain. Australas J Dermatol. 2018;59(4):e269-72.

14. Navadgi S, Pandanaboyana S, Windsor JA. Surgery for Acute Pancreatitis. Indian J Surg. 2015;77(5):446-52. 